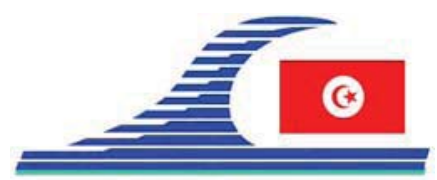

Conférence Méditerranéenne Côtière et Maritime

EDITION 1, HAMMAMET, TUNISIE (2009)

Coastal and Maritime Mediterranean Conference

Disponible en ligne-http://www.paralia.fr - Available online

\title{
Guide Enrochement, traduction française du Rock Manual
}

\section{Céline TRMAL ${ }^{1}$}

1. CETMEF, Division Ouvrages Portuaires Maritimes,

2 Bd du Président Kennedy - BP 5, 13092 Aix en Provence, France.

Celine.trmal@developpement-durable.gouv.fr

\section{Résumé :}

Le guide sur l'utilisation des enrochements dans les ouvrages hydrauliques, appelé Guide Enrochement, vient de paraître en langue française. Ce guide est une traduction de la nouvelle mise à jour du Rock Manual sortie en 2007. Cette mise à jour importante, a été menée par une équipe européenne (Royaume-Uni, Pays-Bas et France), de manière à intégrer les derniers travaux de recherches, le nouveau contexte normatif européen et des nouvelles considérations. Cet article présente l'équipe projet, une vue d'ensemble du contenu du guide et les principaux points qui font l'originalité de ce guide.

\section{Mots-clés :}

Enrochement - Dimensionnement - Ouvrages portuaires - Ouvrages côtiers - Guide technique - Bonne pratique

\section{Introduction}

L'enrochement naturel a toujours été un matériau très utilisé pour la construction des ouvrages hydrauliques de protection contre la houle et les courants de par sa grande disponibilité et son utilisation polyvalente. Dans les années 90, des efforts importants ont abouti à la production de plusieurs guides :

- Le guide technique Les enrochements (LCPC, 1989) publié par le Laboratoire Central des Ponts Chaussées en collaboration avec le Centre d'Etudes Techniques Maritimes et Fluviales, se concentrant sur l'aspect matériaux, a été le guide de base pour la spécification et la fourniture en enrochements naturels pour de nombreux projets français.

- La 1ère édition du Manual on the use of rock in coastal and shoreline engineering (CIRIA/CUR, 1991) a été produite en 1991 résultant d'une collaboration entre le CIRIA (Construction Industry Research and Information Association, Royaume-Uni) et le CUR (Centre for Civil Engineering Research and Codes, Pays-Bas). Ce guide a eu un succès important dans les pays anglophones. Il s'intéresse à la conception, au dimensionnement et aux matériaux naturels dans le contexte de la construction d'ouvrages marins ou côtiers en enrochement. Une édition élargie au domaine des ouvrages en rivières et canaux ainsi qu'au barrage en enrochement a été publiée par le CUR en 1995 DOI: $10.5150 / \mathrm{cmcm} .2009 .083-6$ 
sous le titre de Manual on the use of rock in hydraulic engineering (CUR/RWS, 1995), plus connu sous le nom de Rock Manual.

- D'autres publications sur les digues, endiguements et autres ouvrages en enrochements existent dont notamment le Shore Protection Manual (USACE, 1984) du USACE (United States Army Corps of Engineers, Etats-Unis) mis à jour et réédité sous le nom du Coastal Engineering Manual (USACE, 2003) en 2003.

L'actualisation du guide Rock Manual, qui a débuté en 2003, a été l'occasion pour la France de se doter d'une version en langue française d'un guide de notoriété internationale et également de partager avec les pays francophones un guide complet et à la pointe des nouvelles recherches et recommandations.

\section{L'équipe projet}

La nouvelle édition anglaise du Rock Manual a été le fruit d'une collaboration entre le Royaume-Uni, la France et les Pays-Bas. L'équipe projet anglaise, française et hollandaise était composée respectivement par le CIRIA, CETMEF et le CUR. Les principaux partenaires ont été, au Royaume-Uni, HR Wallingford, Imperial College, Halcrow et l'Université de Southampton, au Pays-Bas, Rijkswaterstaat, Delft Hydraulics, TU Delf, Royal Boskalis Westminster, et en France, CETMEF, CETE de Lyon, SOGREAH, EDF, LCPC.

Elle a aussi bénéficié d'une relecture internationale de manière à assurer que ce guide donne des recommandations sur les pratiques mondiales en cours.

\section{Le contenu}

Le guide est autoporteur dans la mesure où il couvre la conception, le dimensionnement, la construction et la maintenance des ouvrages hydrauliques concernés ainsi que les matériaux de construction, ie les enrochements et les solutions alternatives proches à savoir les blocs artificiels en béton, les matériaux recyclés et les gabions.

Le guide comprend des résultats de recherches récentes, des informations techniques ainsi que des expériences pratiques acquises par les différents professionnels et décrit les bonnes pratiques actuelles. Un effort particulier a été fait pour indiquer les limitations actuelles dans la compréhension des phénomènes physiques complexes en jeux ainsi que la complémentarité des méthodes de conception, des formules empiriques et du jugement de l'ingénieur. Le guide accentue la nécessité de prendre en compte la gestion de l'ouvrage tout au long de son cycle de vie. Les facteurs, autres que techniques qui doivent être pris en compte, par exemple les facteurs sociaux, environnementaux ou économiques sont aussi présentés.

Le guide traite des enrochements dans les ouvrages portuaires, côtiers, estuariens et fluviaux. Dans le domaine portuaire et côtier, tous les outils et recommandations pour le 
dimensionnement, la conception, la construction et l'entretien sont détaillées et ce pour les ouvrages suivants :

- digues à talus, à berme

- ouvrages portuaires (par ex. protections de quais)

- ouvrages côtiers (revêtements, épis, brise-lames, protections anti-affouillement)

- ouvrages offshore (protection des conduites et des câbles, des ouvrages offshore).

\section{L'originalité du guide}

L'originalité du guide tient au fait que toutes les dernières recherches ou méthodes y sont présentées :

- La norme européenne EN 13383 (AFNOR, 2003a; AFNOR, 2003b) sur les enrochements ainsi que toutes les nouvelles pratiques qu'elle implique

- Les nouvelles recherches sur l'intégrité des enrochements, sur le placement et la densité de pose des enrochements

- Une réactualisation des recommandations sur le franchissement des ouvrages par la houle et sur la transmission de la houle

- Une réactualisation des recommandations sur la distribution des hauteurs de houle en eaux peu profondes

- Des recommandations réactualisées sur le dimensionnement géotechnique en faisant référence aux Eurocodes

- Des nouvelles recommandations pour le calcul des forces de la houle sur le mur de couronnement

- Des nouvelles recommandations sur la stabilité du talus arrière des ouvrages en enrochements

- Des nouvelles recommandations sur la stabilité des massifs d'enrochements situés près du fond

- Des recommandations réactualisées sur la stabilité des carapaces en eaux peu profondes

- Des nouvelles recommandations pour la conception et la construction des digues à berme stable statiquement

- Deux nouvelles sections sur le comportement des ouvrages soumis à l'action de la glace.

\section{La disponibilité}

Le Guide Enrochement est distribué par le CETMEF. Pour plus de détails consulter le site :

http://www.cetmef.developpement-durable.gouv.fr/portuaire/manuel-sur-l-utilisationdes-enrochements.html 
Meilleures pratiques environnementales en ingénierie côtière et maritime

\section{Références bibliographiques}

AFNOR (2003a). NF EN 13383-1 Enrochements - Partie 1 : Spécifications, Indice de classement : P18-661-1, $45 \mathrm{p}$.

AFNOR (2003b). NF EN 13383-2 Enrochements - Partie 2 : Méthodes d'essai, Indice de classement : P18-661-2, 44 p.

CIRIA/CUR (1991). Manual on the use of rock in coastal and shoreline engineering. CIRIA special publication 83, CIRIA, 607 p.

CIRIA/CUR/CETMEF (2009). Guide Enrochement. Utilisation des enrochements dans les ouvrages hydrauliques. Traduction française du Rock Manual, PM09.01, CETMEF, 1302 p. Disponible en ligne sur URL :

[http://www.cetmef.developpement-durable.gouv.fr/uploads/RockManual/intro.pdf ]

CUR/RWS (1995). Manual on the use of rock in hydraulic engineering. Report 169, AA Balkema, $800 \mathrm{p}$.

LCPC (1989). Les enrochements. Ministères de l'Equipement, LCPC, 106 p.

USACE (1984). Shore protection manual, 4th edition.

USACE (2003). Coastal Engineering Manual (disponible en ligne sur le site : http://chl.erdc.usace.army.mil) 